\title{
Solvent Extraction Separation of Mo and Co from Chloride Solution Containing Al
}

\author{
Raju Banda ${ }^{1}$, Seong Ho Sohn ${ }^{2}$ and Man Seung Lee ${ }^{1, *}$ \\ ${ }^{1}$ Department of Advanced Material Science \& Engineering, Mokpo National University, Chonnam 534-729, Korea \\ ${ }^{2}$ Korea Institute of Industrial Technology, Incheon Technology Service Centre, 7-47, Songdo-dong, Incheon 406-840, Korea
}

\begin{abstract}
Solvent extraction studies were carried out to investigate the selectivity of some extractants towards Mo and Co from synthetic acidic chloride leach solution of spent HDS catalyst. The composition of the synthetic solution was (in ppm): Mo-394, Co-119, Al-1782 and $3 \mathrm{kmol} / \mathrm{m}^{3}$ of $\mathrm{HCl}$. Among the several solvating and amine extractants that were tested, TOPO and Alamine 308 showed some potential towards the extraction of Mo and Co, respectively. The reasons for this greater extraction tendency of the corresponding extractants were also described thoroughly. Furthermore, the separation of $\mathrm{Co}$ and $\mathrm{Al}$ was investigated by varying the concentration of $\mathrm{HCl}$. Finally, a possible extraction system for the separation of Mo and Co was presented. Our results can be utilized in developing a process for the recovery of Mo and Co from spent catalysts. [doi:10.2320/matertrans.M2012287]
\end{abstract}

(Received August 22, 2012; Accepted October 5, 2012; Published November 16, 2012)

Keywords: cobalt, molybdenum, aluminum, solvent extraction, catalyst

\section{Introduction}

It is well known that the catalysts are indispensable in the petrochemical industry for production of jet fuels, diesel fuels, heavy oil hydrocarbons and gasoline. Hydrodesulphurization (HDS) process has been widely used in petroleum refining industries for removal of sulfur contents from crude oil feed. ${ }^{1)}$ During hydro treating process, catalysts become contaminated with impurities in the crude oil feed stock ${ }^{1)}$ and are deactivated. When this happens, the deactivated catalysts are usually sent for regeneration process due to environmental concerns and recovery of valuable metals.

In order to develop a process to recover Co and Mo from spent HDS catalysts, hydrochloric acid leaching followed by solvent extraction experiments have been tried in our laboratory. From these experimental results, an important feature was observed that the Aliquat 336 showed selective extraction of Mo, when compared with Alamine 336. Apart from amine based extractants, solvating extractants like TBP, TOPO also extracted $\mathrm{Mo}^{2,3)}$ selectively from $\mathrm{Co}$ and $\mathrm{Al}$ and TOPO was found to be better than TBP due to its degradation by acidic chloride solutions. ${ }^{4}$ In fact, the extraction efficiency of $\mathrm{Co}$ (II) with amine based extractants increased with the increase in the concentration of $\mathrm{HCl}$ in aqueous medium. ${ }^{5-8)}$ Additionally, it has been reported that tertiary amines are more efficient to extract metals than other secondary and primary amines ${ }^{9-11)}$ and these primary and secondary amines were not tried in this study.

In the present work, Mo and Co solvent extraction studies were conducted by different amine based and solvating extractants to analyze the extraction behavior and selectivity of metal ions from aqueous chloride solution containing $\mathrm{Al}$ at $3 \mathrm{M} \mathrm{HCl}$. The composition of present work is similar to leach liquor generated by the processing of spent hydrodesulphurization (HDS) catalyst obtained from Korean refineries. The spent HDS catalyst leaching procedure and the optimized leaching conditions have been described in our recent

${ }^{*}$ Corresponding author, E-mail: mslee@mokpo.ac.kr publication. ${ }^{12)}$ The extraction behavior of Mo and Co by amine and solvating extractants was investigated to separate Mo and Co from the synthetic solution. The extraction tendency of tertiary and quaternary amine based extractants for Co was obtained and explained on the basis of their structure. Experimental results reveal that Alamine 308 and TOPO extractants had some affinity for the selective extraction of Co and Mo, respectively.

\section{Experimental}

\subsection{Reagents and procedure}

TBP (Tri butyl phosphate, Yakuri pure chemical Co., Ltd.), TOPO (trioctyphosphine oxide, Sigma-Aldrich), Alamine 300 (trioctyl amine, Sam Chun pure Chemical Co., Ltd.), Aliquat 336 (Tricaprylmethylammonium chloride, a quaternary ammonium salt) Alamine 308 (tri-isooctyl amine), Alamine 336 (mixture of tri-octyl/decyl amine) were purchased from Cognis corporation and used without further purification. All these extractants were diluted with Escaid 110. Decanol (Acros Organics) was used as a modifier.

The synthetic aqueous feed solution containing Mo, Co and $\mathrm{Al}$ was prepared from $\mathrm{Na}_{2} \mathrm{MoO}_{4}$ (Sigma Aldrich), $\mathrm{CoCl}_{2} \cdot 6 \mathrm{H}_{2} \mathrm{O}$ (Junsei Chemical Co., Ltd.) and $\mathrm{AlCl}_{3}$ (Junsei Chemical Co., Ltd.). These reagents were dissolved in analytical grade $3 \mathrm{kmol} / \mathrm{m}^{3} \mathrm{HCl}$ solution. In our present experiments, the composition of the mixed chloride solution was fixed at ppm: Mo-394, Co-119, Al-1782 and $3 \mathrm{kmol} / \mathrm{m}^{3}$ $\mathrm{HCl}^{12)}$

The general extraction experiments were performed by shaking equal volumes of aqueous and organic phases in screwed cap bottles for $5 \mathrm{~min}$ (initial experiments showed that equilibrium was reached within $1 \mathrm{~min}$ ) with wrist action mechanical shaker (Burrell, USA). After shaking experiments, the two phases were separated clearly after $1 \mathrm{~min}$ period of time in all extraction experiments except for the extraction of Co with the tertiary amine based system. It is necessary to describe here that after allowing $10 \mathrm{~min}$ period of time, the two phases were completely separated after 
extraction of $\mathrm{Co}$ from $\mathrm{Al}$ with the tertiary amine based extractant system at $3 \mathrm{kmol} / \mathrm{m}^{3} \mathrm{HCl}$. The concentration of metals in the aqueous phase, before and after extraction, was measured by spectrophotometrically (ICP-OES) and metal contents in the organic phase were determined by mass balance. All the experiments were conducted in duplicate at ambient temperature $\left(25 \pm 1^{\circ} \mathrm{C}\right)$ and the errors associated with the extraction percentage of metals varied within $\pm 5 \%$. During initial extraction tests involved on the extraction of Co with amine based extractants, a kind of third phase was observed in the organic phase. Therefore, $2 \%$ decanol was added as a modifier to prevent the formation of a third phase in organic solution.

\section{Results and Discussion}

\subsection{Effect of amine based extractant concentration on extraction of metals}

The effect of the concentration of amine based extractants (Alamine 336 and Aliquat 336) on extraction of Mo, Co and $\mathrm{Al}$ at $3 \mathrm{kmol} / \mathrm{m}^{3} \mathrm{HCl}$ was tested. The obtained results clearly showed that the extraction percent of Mo and Co are regularly increased with increasing Alamine 336 concentration up to 0.1 for Mo and $0.5 \mathrm{kmol} / \mathrm{m}^{3}$ for Co (see Fig. 1), thereafter reaching quantitative and $50 \%$ extraction efficiency of Mo and Co, respectively. Beyond the 0.1 and $0.5 \mathrm{kmol} / \mathrm{m}^{3}$ Alamine 336, it was noticed that the extraction efficiency of Mo and Co was constant up to $1 \mathrm{kmol} / \mathrm{m}^{3}$. Even in higher concentration of Alamine 336, the extraction efficiency of Co was about $50 \%$. The reason for partial extraction efficiency of cobalt may be ascribed to preferential extraction of $\mathrm{HCl}$ over $\mathrm{Co}$ and $\mathrm{Al}$. This preferential extraction of $\mathrm{HCl}$ over $\mathrm{Co}$ with several amine based extractants has already been reported in aqueous hydrochloric acid and its mixture with $\mathrm{LiCl}$ solutions. ${ }^{7,13)}$ Moreover, it can obviously be concluded that the extraction efficiency of $\mathrm{Al}$ was nil in all studied concentration range of Alamine 336.

In order to get selective separation of Mo from $\mathrm{Co}$ and $\mathrm{Al}$, the effect of Aliquat 336 concentration was also tested. The obtained results are demonstrated in Fig. 2. Under our experimental conditions, the percent extraction of Mo and Co increased uniformly from 0.9 to 97.6 and 0 to 11.2 with increasing Aliquat 336 concentration from 0.001 to $1 \mathrm{kmol} / \mathrm{m}^{3}$, whereas the percent extraction of $\mathrm{Al}$ was nil in our concentration range.

Figures 1 and 2 indicate that the co-extraction of $\mathrm{Co}$ with Aliquat 336 was lesser than that with Alamine 336. It has been reported earlier that Aliquat 336 can extract only anionic species of cobalt from aqueous chloride solutions. ${ }^{14-16)}$ Furthermore, Aliqaut 336 is known to have a greater extraction efficiency towards $\mathrm{HCl}$ when compared with the other amine based extractants. ${ }^{7,13)}$ Eventually, from these explanations and obtained results in the present experiments, we may expect that the reason for lower extraction efficiency of Co with Aliquat 336 is due to its chloride form having much weaker complexes with cobalt. ${ }^{17)}$ It is important to mention that, during the preparation of Aliquat 336 solution in Escaid 110 diluent, it was observed that two phases were formed in the organic phase when the concentration of Aliquat 336 was $0.5 \mathrm{kmol} / \mathrm{m}^{3}$. The

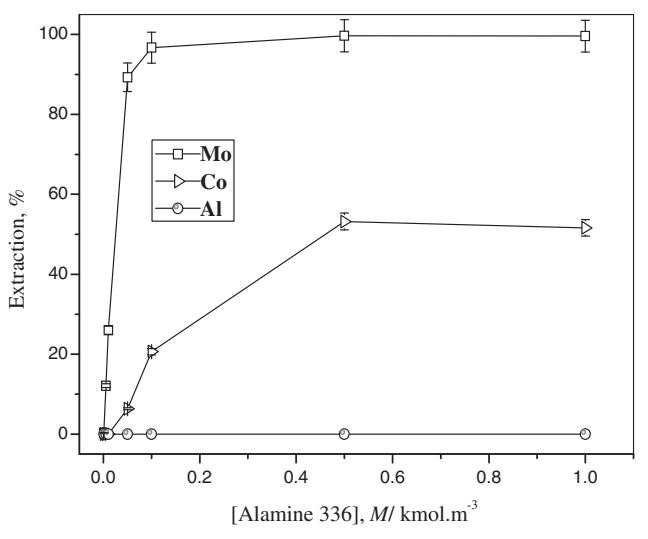

Fig. 1 Effect of Alamine 336 concentration on extraction of Mo, Co and $\mathrm{Al}$.

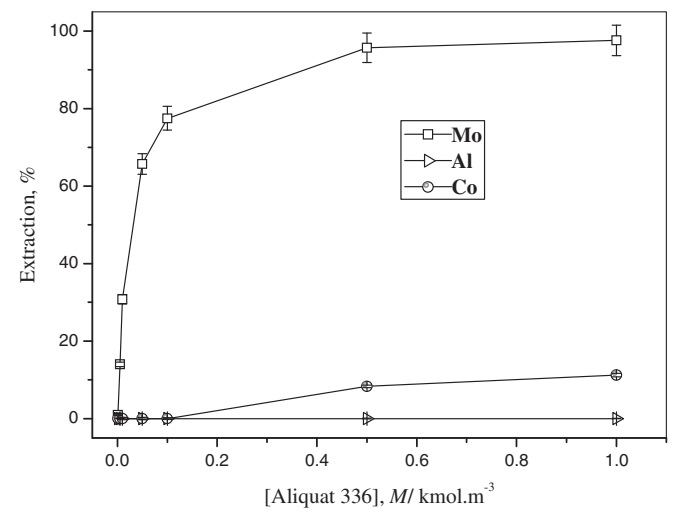

Fig. 2 Effect of Aliquat 336 concentration on extraction of Mo, Co and Al.

formation of two phases in organic phase is due to fact that the exctractant is not dissolving in Escaid 110 diluent properly, which indicates that Escaid 110 is not a suitable diluent for Aliquat 336 in higher concentration range. However, diluents can also play a great role on the extraction of cobalt with amine based extractant system. ${ }^{18)}$

\subsection{Effect of TBP and TOPO concentration on extrac- tion of metals}

In order to get detailed information on the separation of Mo from $\mathrm{Co}$ and $\mathrm{Al}$, some additional experiments were also conducted by using TBP and TOPO. As the concentration of TBP and TOPO increased, the percent extraction of Mo increased (see Figs. 3 and 4) uniformly and it became quantitative at around $0.5 \mathrm{M}$ and $0.05 \mathrm{kmol} / \mathrm{m}^{3}$, respectively, whereas the percent extraction of $\mathrm{Co}$ and $\mathrm{Al}$ was nil. Comparing the extraction system between TBP and TOPO, it is apparent that the extraction efficiency of Mo with TOPO is higher than that of TBP. The reason for the difference in extraction efficiency of TOPO and TBP is due to the fact that TOPO has electron donating groups while TBP has electron withdrawing groups.

Among the studied extractant systems, TOPO shows some potential to separate Mo selectively from $\mathrm{Co}$ and $\mathrm{Al}$ and TOPO was selected as a good extractant to separate Mo from acidic chloride solution containing Co and Al. In terms of stripping, some studies have reported that stripping of metal 


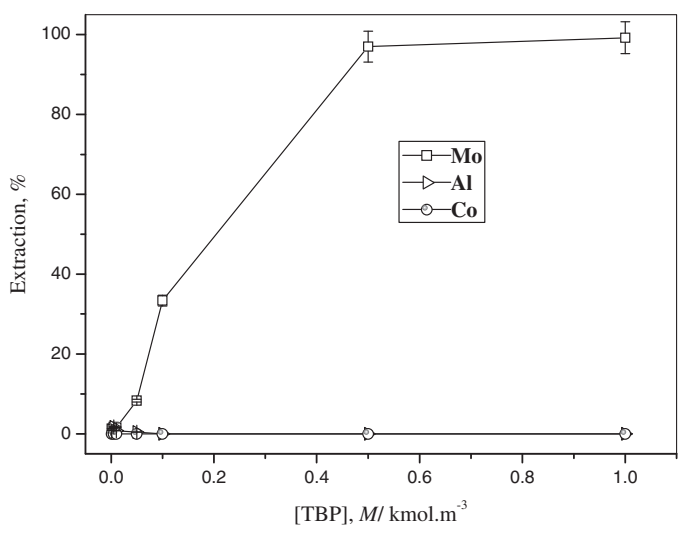

Fig. 3 Effect of TBP concentration on extraction of Mo, Co and Al.

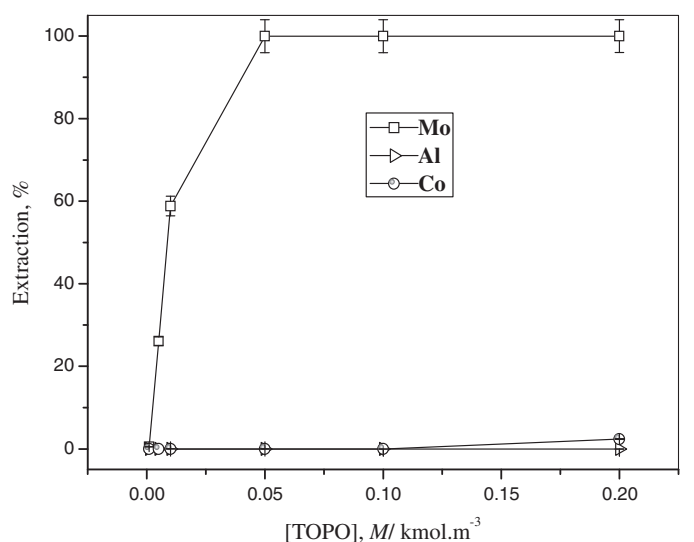

Fig. 4 Effect of TOPO concentration on extraction of Mo, Co and Al.

from the loaded TBP is easier than that from the loaded TOPO. ${ }^{19,20)}$ Therefore, selectivity for Mo together with the ease of stripping should be considered simultaneously in choosing an extractant for the separation of Mo from Co and $\mathrm{Al}$.

\subsection{Effect of Aliquat 336, Alamine 300, Alamine 336 and} Alamine 308 concentration on extraction of cobalt

The selectivity experiments involving several extractants, such as, Aliquat 336, Alamine 300, Alamine 336 and Alamine 308 were carried out by varying their concentration on extraction of $\mathrm{Co}$ from Mo free raffinate containing $\mathrm{Al}$ at $3 \mathrm{kmol} / \mathrm{m}^{3} \mathrm{HCl}$. The obtained experimental results are depicted in Fig. 5. As is shown from Aliquat 336 extraction system (see Fig. 5), the extraction of Co increased slightly from 0 to $\sim 20 \%$ with increasing Aliquat 336 concentration, whereas the percent extraction of $\mathrm{Al}$ was nil under the same experimental conditions. The exhibited poor extraction efficiency of Co may result from preferential extraction of $\mathrm{HCl}$ over $\mathrm{Co}$ and $\mathrm{Al}^{7)}$ Compared to the extraction of cobalt in the presence of $\mathrm{Mo}$, the extraction percentage of Co was slightly increased in the absence of Mo at the same concentration of Aliquat 336.

To evaluate extraction performance of Co with different extractants, an extraction experiment was carried out by varying Alamine 300 concentration at $3 \mathrm{kmol} / \mathrm{m}^{3} \mathrm{HCl}$ aqueous chloride solution containing Al. The results (see

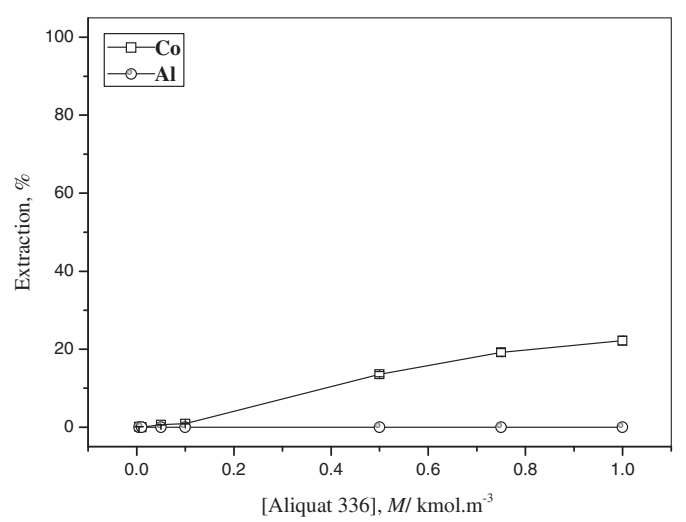

Fig. 5 Effect of Aliquat 336 concentration on extraction of Co and Al.

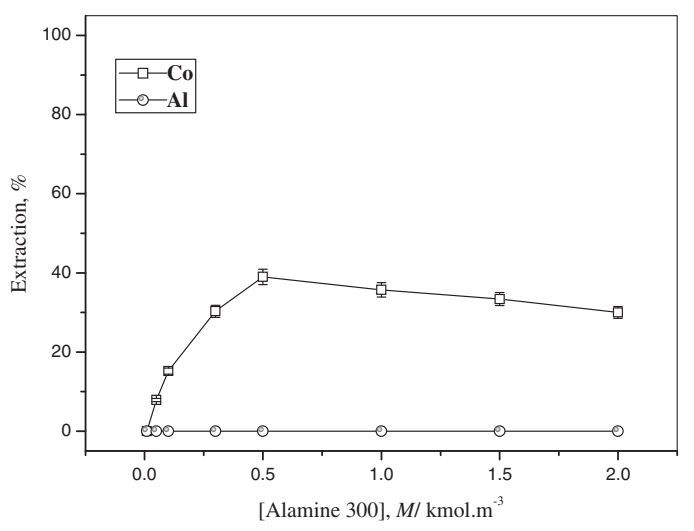

Fig. 6 Effect of Alamine 300 concentration on extraction of Co and Al.

Fig. 6) showed that the extraction efficiency of cobalt increased from 0 to $39 \%$ with increasing Alamine 300 concentration up to $0.5 \mathrm{kmol} / \mathrm{m}^{3}$ and afterwards it decreased slightly from 39 to $31 \%$. The reason for the decrease of Co extraction with increasing Alamine 300 concentration may be due to the preferential extraction of $\mathrm{HCl}$ over $\mathrm{Co}$ and $\mathrm{Al}$.

Additionally a few experiments were also conducted regarding the extraction behavior of Co with Alamine 336 and Alamine 308 at $3 \mathrm{kmol} / \mathrm{m}^{3} \mathrm{HCl}$. It can be seen from Figs. 7 and 8 that, the selective extraction of Co increased from 0 to $52.7 \%$ and 0 to $72.4 \%$ with increasing Alamine 336 and Alamine 308 up to $0.5 \mathrm{kmol} / \mathrm{m}^{3}$ concentration, thereafter the extraction percent of Co decreased with increasing Alamine 336 and Alamine 308 concentration. The decrease in the extraction percent of $\mathrm{Co}$ at higher concentration of Alamine 336 and Alamine 308 may be related to the preferential extraction of $\mathrm{HCl}$ over $\mathrm{Co}$ into the organic phase. ${ }^{7,13)}$

Figure 5 clearly shows that extraction percentage of Co by Aliquat 336 was lower than that by other tested tertiary amine based extractants. The reason for this difference of Co extraction behavior by tertiary and quaternary amines lies in the fact that quaternary amines have a greater extraction tendency towards $\mathrm{HCl}$ compared with the tertiary amines. ${ }^{7)}$ Hence, our results agreed well with the reported data in that the tertiary amine extractants exhibited greater extraction tendency towards Co than Aliquat 336. 


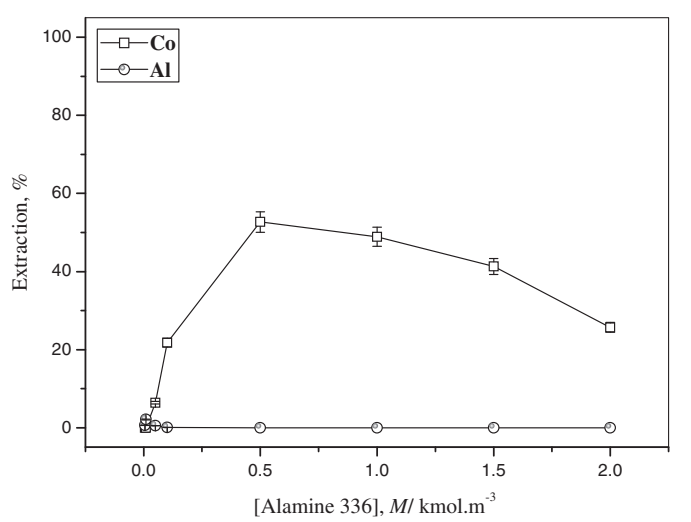

Fig. 7 Effect of Alamine 336 concentration on extraction of Co and Al.

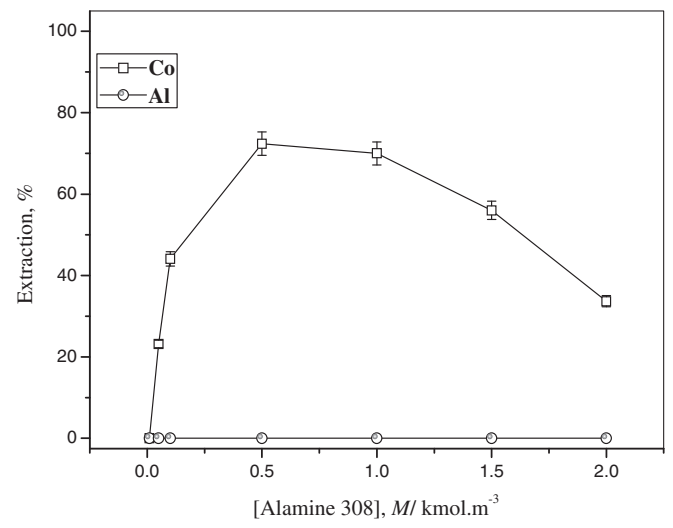

Fig. 8 Effect of Alamine 308 concentration on extraction of Co and Al.

Moreover, it is significant to highlight here that Alamine 308 exhibits better extraction capability towards Co than Alamine 336 and Alamine 300 under similar experimental conditions. Therefore from the above statement, it may be concluded that the structure of Alamine 308 (the space between the molecules is longer) extractant may determine its affinity towards Co extraction.

After equilibrium experiments were conducted with Alamine 300, Alamine 336 and Alamine 308 extractant for selective separation of $\mathrm{Co}$ from $\mathrm{Al}$ at $3 \mathrm{kmol} / \mathrm{m}^{3} \mathrm{HCl}$, the disengagement of the two phases were perfectly clear after 10 min period of time and a sort of third phase was observed. Furthermore, it was detected that the organic phase was light bluish color and viscosity of organic solution.

\subsection{Effect of hydrochloric acid concentration on the extraction of cobalt in the presence of constant concentration of $\mathrm{Al}$}

An experiment was designed to investigate the extraction behavior of $\mathrm{Co}$ with increasing $\mathrm{HCl}$ concentration from 3 to $5 \mathrm{kmol} / \mathrm{m}^{3}$ in the presence of constant proportion of $\mathrm{AlCl}_{3}$ and Alamine $308\left(0.5 \mathrm{kmol} / \mathrm{m}^{3}\right)$. The obtained experimental results (Fig. 9) showed that the extraction of Co increased from 72.4 to $99.1 \%$ with increasing $\mathrm{HCl}$ concentration from 3 to $5 \mathrm{kmol} / \mathrm{m}^{3}$, whereas, extraction of $\mathrm{Al}$ was nil in the studied concentration range.

Eventually, our solvent extraction studies reveals that Alamine 308 extractant showed a good extraction perform-

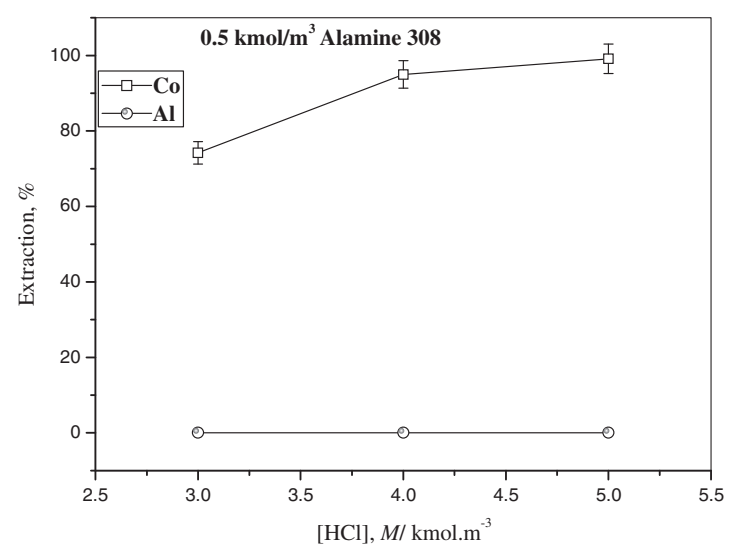

Fig. 9 Effect of $\mathrm{HCl}$ concentration on extraction of $\mathrm{Co}$ and $\mathrm{Al}$.

ance towards Co from Al selectively and that Alamine 308 was found to be more effective in separating Co than other studied extractants, such as Aliquat 336, Alamine 300 and Alamine 336. Therefore it can generally be concluded that the Alamine 308 extractant system is suitable to separate Co from aqueous chloride solution containing Al.

\section{Conclusions}

In order to recover Mo and Co from the synthetic chloride leach solution of spent HDS catalysts, solvent extraction experiments by amine based and solvating extractants have been performed. The chemical composition of our synthetic solution was Mo-394 ppm, Co-119 ppm, Al-1782 ppm and $3 \mathrm{kmol} / \mathrm{m}^{3} \mathrm{HCl}$. Among the all studied extractants, TOPO showed better extraction capability towards Mo from Co and $\mathrm{Al}$ at $3 \mathrm{kmol} / \mathrm{m}^{3} \mathrm{HCl}$ solution. Alamine 308 was found to be the best extractant for Co from Mo free raffinate at $3 \mathrm{kmol} / \mathrm{m}^{3} \mathrm{HCl}$ solution. Cobalt can be extracted from the Mo free raffinate by increasing the concentration of $\mathrm{HCl}$ with Alamine 308. The extraction behavior of Co by tertiary and quaternary amines was compared on the basis of their structure.

\section{Acknowledgement}

This work was financially supported by a grant from the fundamental R\&D Program for Core Technology of Materials funded by the Ministry of Knowledge Economy, Republic of Korea.

\section{REFERENCES}

1) B. W. Jong, S. C. Rhoads, A. M. Stubbs and T. R. Stoelting: Recovery of principal metal values from waste hydroprocessing catalysts, (US Bureau of Mines, US Department of Interior, RI 9252, August 1989).

2) T. Sato, H. Watanabe and H. Suzuki: Hydrometallurgy 23 (1990) 297308.

3) P. Tkac and A. Paulenova: Sep. Sci. Technol. 43 (2008) 2641-2657.

4) G. Haesbroek, A. De Schepper and A. Van Peteghem: Extractive Metall. Nickel Cobalt, Proc. Symp., 117th TMS Annual Meeting, (1998) p. 463.

5) M. Filiz, N. A. Sayar and A. A. Sayar: Hydrometallurgy 81 (2006) $167-173$. 
6) N. A. Sayar, M. Filiz and A. A. Sayar: Hydrometallurgy 96 (2009) $148-153$.

7) M. L. Good, S. E. Bryan, F. F. Holland, Jr. and G. J. Maus: J. Inorg. Nucl. Chem. 25 (1963) 1167-1173.

8) T. Sato, T. Nakamura and T. Fujimatsu: Bull. Chem. Soc. Jpn. 54 (1981) 2656-2661.

9) N. A. Sayar, M. Filiz and A. A. Sayar: Hydrometallurgy 96 (2009) $148-153$.

10) N. I. Gerhardt, A. A. Palant, V. A. Petrova and R. K. Tagirov: Hydrometallurgy 60 (2001) 1-5.

11) A. A. Palant, N. A. Iatsenko and V. A. Petrova: Hydrometallurgy 48 (1998) 83-90.

12) B. Raju, S. H. Sohn and M. S. Lee: J. Hazard. Mater. 213-214 (2012)
$1-6$.

13) S. Kagaya, R. W. Cattrall and S. D. Kolev: Anal. Sci. 27 (2011) 653657.

14) B. Wassink, D. Dreisinger and J. Howard: Hydrometallurgy 57 (2000) 235-252.

15) T. Sato, S. Shimomura, T. Murakami, T. Maeda and T. Nakamura: Hydrometallurgy 12 (1984) 245-254.

16) H. C. Kao and R. S. Juang: J. Membr. Sci. 264 (2005) 104-112.

17) T. Sato, K. Adachi, T. Kato and T. Nakamura: Sep. Sci. Technol. 17 (1982) $1565-1576$

18) M. L. Good and S. E. Bryan: J. Inorg. Nucl. Chem. 20 (1961) 140-146.

19) K. C. Sole: Hydrometallurgy 51 (1999) 239-253.

20) K. C. Sole: Hydrometallurgy 51 (1999) 263-274. 